\title{
Reduction of Penstock Fatigue in a Medium-Head Hydropower Plant Providing Primary Frequency Control
}

\author{
Stefano Cassano \\ PERSEE, Mines ParisTech - PSL Univ. \\ Sophia Antipolis, France \\ stefano.cassano@mines-paristech.fr
}

\author{
Christophe Nicolet \\ Power Vision Engineering Sàrl \\ St-Sulpice, Switzerland \\ christophe.nicolet@powervision-eng.ch
}

\author{
Fabrizio Sossan \\ PERSEE, Mines ParisTech - PSL Univ. \\ Sophia Antipolis, France \\ fabrizio.sossan@mines-paristech.fr
}

\begin{abstract}
The displacement of conventional electricity generation in favor of stochastic renewables will require extended regulation capacity from remaining dispatchable plants, including hydropower power plants (HPPs), which may be subject to accelerated aging of its mechanical parts due to more severe regulation duties. This paper focuses on transient pressures in the penstock of medium-head HPP due to load variations from primary frequency control. We propose and compare the performance of two algorithms to reduce pressure transients, ultimately leading to decreased penstock fatigue. The first is a linear filter applied to the input control signal of the HPP. The second, that we call fatigue-aware, uses a simplified model of the HPP to estimate the fatigue of the penstock and selectively remove those patterns from the input control signal that are conducive to accelerate fatigue damage. The performance of the two algorithms is validated by simulations considering a $230 \mathrm{MW}$ medium-head HPP.
\end{abstract}

Index Terms-Hydro power, Hydraulic Transient, Wear and tear, Primary frequency control (PFC), Filter

\section{INTRODUCTION}

Several countries worldwide have set out ambitious energy transition targets to increase the deployment of renewable generation and decommission fossil-fuel and nuclear power plants at the end of their service life. Today, conventional power plants are responsible for providing the majority of grid ancillary services. Their displacement in favor of nondispatchable resources, like stochastic renewables, will require to identify new providers of ancillary services. This need has also been recognized at the level of the European Union, which has supported in the recent years extensive research efforts aimed at investigating and enhancing the flexibility for future power systems, see e.g. the research projects EU-SysFlex, OSMOSE, and XFLEX Hydro [1]-[3].

Hydropower plants (HPPs) already provide essential electricity generation and regulation services (this latter thanks to extended regulation capacity and fast ramping time compared to the other kinds of generation). In the future, they will be

This research was supported by the European Union Horizon 2020 research and innovation program in the context of the Hydropower Extending Power System Flexibility project (XFLEX HYDRO, grant agreement No 857832).

978-1-7281-1078-3/20/\$31.00 (C)2020 IEEE called to play an even more prominent role. Indeed, integrating a larger proportion of stochastic renewable generation will increase the need for regulation, with steeper ramping duties and more frequent start-and-stops for conventional generation. Increased flexibility requirements may be conducive to increased levels of wear-and-tear of the mechanical components, ultimately leading to reduced operational efficiency due to higher maintenance costs and reduced plant availability. The work in [4] has shown that, in medium-head HPPs, the provision of secondary and primary frequency control (PFC) increases the level of penstock fatigue. As the penstock is a critical component of these plants, it is important for plant owners to i) plan hardware upgrades to enable profitable operations in future flexibility markets while postponing major infrastructure investments, and, ii) design control policies that maximizes the global flexibility of the system and the service life of all the components.

Hardware upgrades to increase the flexibility of HPPs include variable speed technology and hybridization with battery energy storage systems (BESSs). Variable speed refer to decoupling the speed of the turbine rotor from the grid frequency, thus opening to operate the turbine at higher efficiency, see e.g. [5]. Hybridization refers to adding a battery system in parallel to the HPP. It has two main operational objectives. First, extending the overall regulation capacity of the system, as demonstrated in [6], [7] for the case of hybridization of flexible demand. Second, reducing wear and tear thanks to avoiding operational patterns conducive to large levels of mechanical stress for the HPP components. The use of BESSs is motivated by their decreasing costs [8], excellent ramping capabilities due to the lack of mechanical time constants [9], and modularity, that allows a flexible custom design and to add them as extensions of existing electrical infrastructure. The advantage of installing a BESS at the plant location compared to using distributed BESSs (e.g., [10], [11]) is better situational awareness and more informed decision making thanks to being connected to the SCADA system of the plant. Moreover, thirdparty-owned BESSs in other grid locations would be operated through existing flexibility markets that, being slower than BESS's response time (minutes vs. sub-second), would not 
enable to exploit their operational potential fully.

This paper considers the problem of designing a control algorithm to reduce the cumulative fatigue of a penstock of a medium-head HPP when it provides regulation services to the power grid. A similar problem has been considered in [12] for hydro turbines. In particular, we propose and compare the performance of two algorithms to reduce the penstock fatigue. The first one is a simple low-pass filter that, thanks to smoothing the quick variations of the control set-point, achieves to reduce the pressure variations over time along the penstock, thus reducing the mechanical stress. The second algorithm explicitly models the fatigue cycles by embedding a formulation of the rain-flow counting algorithm for fatigue estimations. It achieves to selectively filter those portions of the control set-point that engender mechanical fatigue in the penstock. By virtue of this property, this algorithm is called fatigue-aware filter. Algorithms' performance is validated by simulations considering a realistic model of an HPP providing PFC to the power grid.

These algorithms can be used in a hybrid HPPs to split a single control signal into multiple components (one per controllable resource) to achieve reduced stress levels in the penstock. However, in this paper, we focus only on the hydraulic components. The design of a suitable control signal for a BESS will be considered in future works.

The rest of this paper is organized as follows: Section 2 describes the problem, Section 3 describes the method, Section 4 describes the case study and simulation models, Section 5 presents the results and Section 6 draws the main conclusions.

\section{Problem Statement}

We consider a medium-head HPP that is controlled to track a frequency set-point, for example the one for PFC. The tracking action is accomplished by the HPP governor (typically a PID controller), which processes the frequency set-point and controls the power output of the turbine by adjusting on the guide vane opening. Large changes in the guide vane opening may cause water hammer and pressure transients along the penstock, that, due to fatigue limitations, might incur in fatigue damage. The problem tackled in this paper is how to adjust the set-point of the turbine to reduce the pressure transients and enhance the service time of the penstock.

\section{METHODS}

\section{A. Linear low-pass filter}

Water hammer determines pressure variations and mechanical stress in the penstock and is caused by abrupt changes of the power set-point. A first intuitive solution to reduce the mechanical stress consists in smoothing the input control signal with a low-pass filter. In this context, the key parameter to be determined is the cut-off frequency of the filter, that is designed as described next.

The authors of [13] describes a power-set-point-to-penstockhead linear transfer function model. It is identified by applying a pseudo-random-binary-signal to a detailed simulation model of the plant. This transfer function points out the natural frequencies of the hydraulic systems. In particular, if we neglect the pressure fluctuation from the mass oscillation period between the surge tank and the upper reservoir which turns in a severe filtering action due to its large periods and, therefore, small frequencies, then the first natural frequency of the penstock features maximum pressure amplitudes at the penstock end. The first natural frequency of the penstock, denoted by $f_{0}$, can be calculated as follows:

$$
f_{0}=\frac{a}{4 L},
$$

where $L$ is the total length of the penstock and $a$ is the average wave speed in the penstock. We use the frequency $f_{0}$ as the cut-off frequency for the first-order linear filter to smooth quick variations of the input control signal of the plant.

\section{B. Fatigue-aware filter}

The linear filter discussed above smooths quick variations of the control signal, thus achieving smaller pressure transients and decreased levels of mechanical stress. Its drawback is that it also filters cycles with virtually no impact on the fatigue (e.g., those with very small amplitude), ultimately leading to poorer tracking performance of the original control signal and increased energy capacity requirements in case of hybrid HPPs. Starting from this intuition, we describe in this subsection a filtering algorithm that, by explicitly modeling the penstock fatigue, finally achieves a more informed filtering action and improved tracking performance. For this reason, we call this filter fatigue-aware. In the rest of this section, we explain, first, how the penstock fatigue is assessed and, later, the filter design.

1) Evaluation of the fatigue: The theory of cumulative damage assumes that a stress cycle with an alternating stress above the endurance cut-off limit inflicts a measurable permanent damage. The total damage caused by a number of stress cycles can be modeled as the summation of damages caused by the individual stress cycles [14]. The penstock fatigue is estimated with the following steps [4]:

(i) evaluation of the pressure time series at different sections of the penstock. For this step, we resort to a simulation model from the existing technical literature described in Section IV.

(ii) pressure is converted into mechanical stress $\sigma$. For openair penstosck, we use the following model [15]:

$$
\sigma(t)=p(t) \frac{D}{2 e}
$$

where $D$ and $e$ are the penstock diameter and wall thickness, respectively;

(iii) cycle counts of the mechanical load of each section of the penstock with a rainflow algorithm, which returns the number of cycles associated to a given stress level [16], [17].

Once the cycle counts and the associated stress levels are calculated, we use the SN diagram, or Wohler curve, to compute the fatigue. Fig. 1 shows the SN diagram with different curves for different welding quality categories according to standard 
BS7910 [18]. Each SN curve reports the maximum number of cycles that the component can undergo ( $\mathrm{x}$-axis) at a given value of the stress (y-axis) before a failure is likely to occur. As visible in Fig. 1, each curve (corresponding to a law of the kind $\Delta \sigma^{3} N=$ constant), features a higher number of cycles for decreasing values of the fatigue limit. However, below a certain stress level, called effective fatigue limit, the number of cycles increases drastically, in other words, cycles below a certain load have a negligible impact on the fatigue. This is the key notion that we are going to leverage in the fatigue-aware filter, as we describe next.

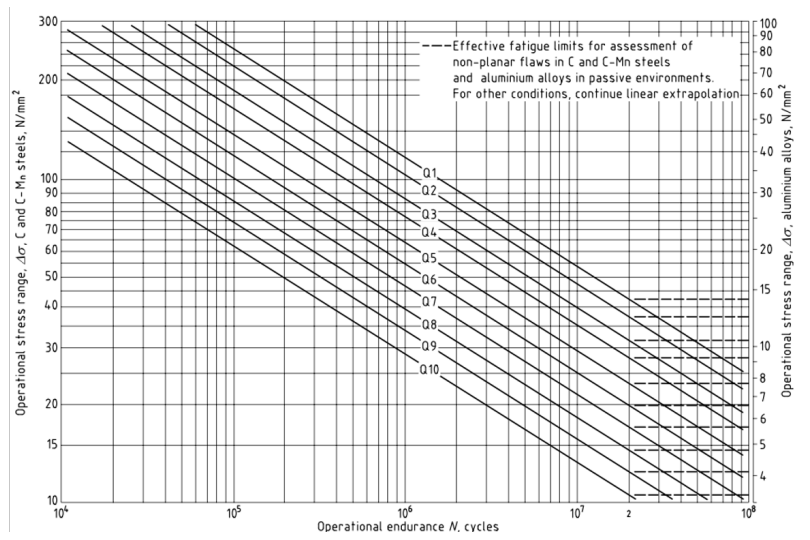

Fig. 1. S-N curves of steel for different welding constructive details [18].

2) Description of the fatigue-aware filter: As described above, stress cycles below the effective fatigue limit threshold do not count towards accumulating relevant penstock fatigue. The intuition behind the aging-aware filter is adjusting the HPP power set-point in order to avoid cycles above the effective fatigue of its penstock. We accomplish this with the algorithmic tool-chain shown in Fig. 2. The input of the system is the HPP's power set-point. An HPP model, $G(s)$ (discussed later), is used to compute the resulting guide vane opening $y$, which is then applied to the model $H(s) / y(s)$ to determine the head at a section of the penstock. In this development, we consider the element of the penstock that it is subject to the largest stress variations, i.e. the 5th element.

The operational requirement that we implement is that stress variations around the nominal value, denoted by $\sigma$, should not conduce to exceed the effective fatigue limit $\bar{\sigma}$. This condition reads as:

$$
\left|\sigma^{*}(t)-\boldsymbol{\sigma}\right| \leq \bar{\sigma}
$$

The value $\bar{\sigma}=11 \mathrm{MPa}$ is extracted from Fig. 1 considering the most conservative quality category (i.e., Q10). The filtered stress $\sigma^{*}$ is calculated by the filter $F$ as a function of the input stress $\sigma(t)$ as:

$$
\sigma^{*}(t)=\boldsymbol{\sigma}+\min (|\sigma(t)-\boldsymbol{\sigma}|, \bar{\sigma}) \cdot \operatorname{sign}(\sigma(t)-\boldsymbol{\sigma}),
$$

which achieves the filtering action shown in Fig. 3. The filtered stress $\sigma^{*}$ is translated to $H^{*}$ by applying the inverse of (2). From the trimmed head $H^{*}$, we apply the inverse transfer function model $y(s) / H(s)$ and the inverse HPP's model $G^{-1}(s)$ to reconstruct the guide vane opening and power set-point, respectively.

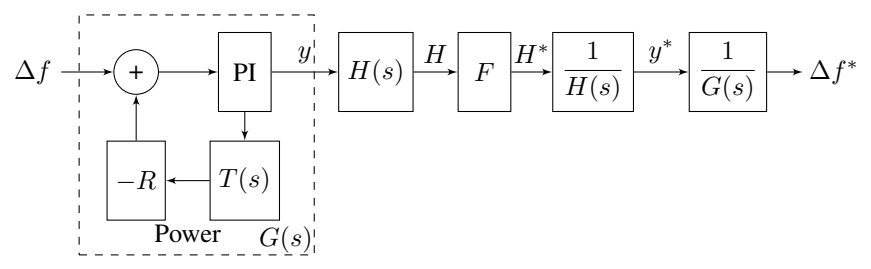

Fig. 2. Fatigue-aware filter. The frequency deviation $\Delta f$ (i.e., frequency setpoint minus the measured frequency) is used to compute guide vane position $y$ and head $H$ in the penstock's most fatigue-critical element. The filtered head is reconverted to $\Delta f^{*}$ by applying the inverse modeling toolchain.

The HPP's model $G(s)$ and guide-vane-to-head $H(s) / y(s)$ are both transfer function reduced-order models chosen because they are invertible. They are from [19]. The reducedorder model $G(s)$ requires a parameter estimation procedure to accurately replicate the behavior of the detailed simulation of the plant due to a different interpretation of the input control signal, that in [19] is referred to as gate opening $g$ and in our model as guide vane $y$. We adopt the quadratic relationship between the two proposed by the authors of [20]

$$
g(y)=d_{0}+d_{1} \cdot y+d_{2} \cdot y^{2} .
$$

where $d_{0}, d_{1}$ and $d_{2}$ are parameters found by minimizing the root mean square deviation among the two models.

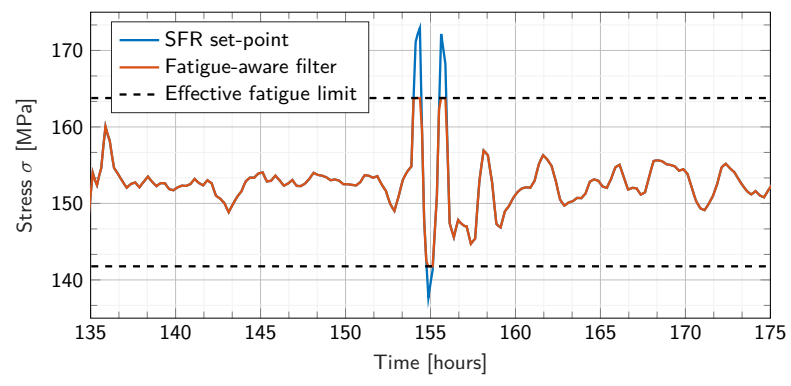

Fig. 3. Effect of the fatigue-aware filter on the stress at the penstock element closest to the turbine. The maximum allowed level of stress variations, chosen considering the effective fatigue limit of the penstock, is set to $11 \mathrm{MPa}$.

\section{CASE STUdy AND HPP MOdel}

We consider a $230 \mathrm{MW}$ medium-head HPP equipped with a Francis turbine. An open-air 1,100-meter-long penstock connects the power house to the upper-level reservoir, that are located at a difference of altitude of 315 meters. The characteristics of the HPP are summarized in Table 1. In the rest of this section, we discuss in detail the components of the simulation models of the HPP, namely the penstock, the turbine, and HPP governor. The HPP considered at this stage does not include a surge tank that will be considered in future works. 
TABLE I

CASE STUDY MODEL PARAMETERS

\begin{tabular}{|l|c|c|}
\hline Parameter & Unit & Value \\
\hline Nominal Power & MW & 230 \\
Number of turbines & MW & 1 \\
Nominal head & $\mathrm{m}$ & 315 \\
Nominal discharge & $\mathrm{m}^{3} / \mathrm{s}$ & 85.3 \\
Nominal speed & $\mathrm{rpm}$ & 375 \\
Nominal torque & $\mathrm{Nm}$ & $5.86 \times 10^{6}$ \\
Length of penstock & $\mathrm{m}$ & 1100 \\
Diameter of penstock & $\mathrm{m}$ & 5 \\
Wave speed & $\mathrm{m} / \mathrm{s}$ & 1100 \\
\hline
\end{tabular}

\section{A. Hydraulic system}

1) Penstock: The penstock model, from [21], is based on mass and momentum conservation laws and accounts for the water elasticity. Being the length of the penstock significantly larger than its diameter, the model assumes that the water flows normally to the penstock cross-section $A$, and its pressure $p$, flow velocity $C$ and density $\rho$ are uniform in the cross-section. The model reads as the following set of partial differential equations:

$$
\left\{\begin{array}{l}
\frac{\partial h}{\partial t}+\frac{a^{2}}{g A} \cdot \frac{\partial Q}{\partial x}=0 \\
\frac{\partial h}{\partial x}+\frac{1}{g A} \cdot \frac{\partial Q}{\partial t}+\frac{\lambda|Q|}{2 g D A^{2}} \cdot Q=0,
\end{array}\right.
$$

where $h$ is the piezometric head, $Q$ the discharge, $\lambda$ the local loss coefficient, $g$ the acceleration of gravity and $a$ the wave speed in $\mathrm{m} / \mathrm{s}$.

The model in (6) is converted to a set of ordinary differential equations by applying a first order spatially-centred Lax discretization scheme, leading to an equivalent electric circuit (EEC) which is solved with the finite difference method. The EEC analogy consists in representing the penstock as a series of T-shaped RLC circuits where voltages are pressures and currents are flows. The EEC model is shown in Fig. 4(b) for the case with one element, where $h_{1+1 / 2}, Q_{1}$, and $Q_{2}$ are the state variables, and $H_{r}$ and $H_{2}$ are input. Its RLC parameters are:

$$
R=\frac{\lambda \cdot|Q| \cdot d x}{2 \cdot g \cdot D \cdot A^{2}}, \quad L=\frac{d x}{g \cdot A}, \quad C=\frac{g \cdot A \cdot d x}{a^{2}},
$$

where $d x$ is the spatial discretization interval.

2) Francis turbine: Francis reaction turbines convert kinetic and potential energy of the fluid into mechanical work. The transient behaviour of the turbine can be accurately simulated by considering the "quasi-static" approach, which assumes that the machine undergoes a succession of different steady-state operating points. This approach ensures tractable computational times with sufficient accuracy for all the flow regimes during transients [21]. The turbine model is added to the equivalent electric circuit of the penstock with the variable voltage (i.e., pressure) source $H_{t}(y, N, Q)$ in Fig. 4(b), that converts the hydraulic energy into mechanical work. The pressure is modeled using the polar representation of the turbine characteristic $W_{H}(y, \theta(Q, N))$ while the mechanical torque

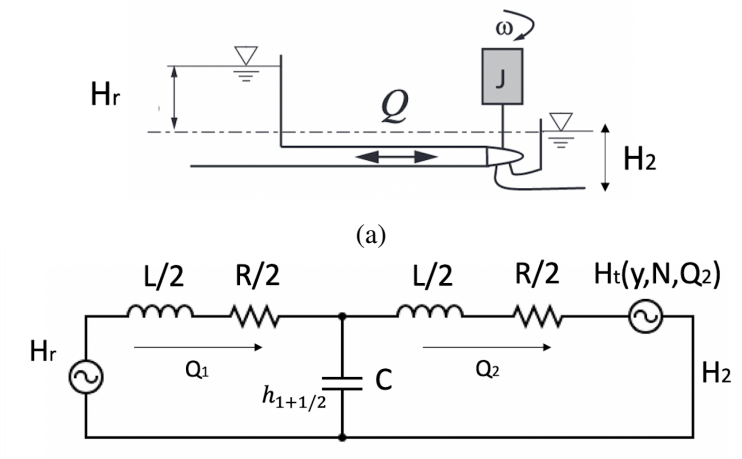

(b)

Fig. 4. Reservoir, penstock, and turbine (a) and equivalent electric circuit (b) considering a one-element penstock model [21].

is obtained from the torque characteristic $W_{B}(y, \theta(Q, N))$. The inertia of the water and the no-discharge condition at guide vane $y$ full closure are respectively modelled with an equivalent inductance and a resistance in series to the turbine model (not shown in Fig. 4 for a reason of space).

3) Complete model and implementation: The EEC model including the turbine is converted to a time variant statespace model with $2 n+1$ equations (where $n$ is number of discretized penstock elements), plus 1 equation for the rotational speed of the turbine. The state variables are the $n$ heads $\left(h_{i+1 / 2}\right)$ at the center of each element $i=1, \ldots, n$, the $n+1$ discharges through the pipe $Q_{i+1}$ and the turbine rotational speed $\omega$. The inputs are the up- and down-stream heads $H_{r}, H_{2}$, the head at the turbine $H_{t}$ and mechanical torque $T_{t}$. The state-space model is implemented in Matlab and solved with fourth order Range-Kutta integration. In order to assure the numerical stability of the integration process, the time step of the simulation should be less than the time the travelling wave takes to move from two discretized points of the space, a condition known as the CFL criteria [21]. The model is validated by simulating water hammer, step response of Francis turbine and emergency shutdown, and comparing them against a state-of-the-art commercial software for HPPs simulations. Validation results are reported in the appendix.

\section{B. HPP governor}

The turbine governor includes a standard proportionalintegral (PI) regulator with droop regulator for $\mathrm{PFC}$, and set-point for speed-changer setting, like in [19]. The PI implements limits on the rate-of-change and magnitude of the output to reproduce the limitations of the guide vane actuators [22]. The permanent speed droop is set to $2.0 \%$. Compared to conventionally adopted droops for HPP (that are in the range $2.5-5 \%$ ), we choose a lower droop to reproduce a future operational context where larger flexibility is required from dispatchable resources. The PI parameters are determined with the Ziegler-Nicholas method [22]. The dynamic performance of the governor is tested by verifying the ENTSOE qualification tests for PFC in Appendix A. 


\section{RESUlTS AND DisCUSSION}

\section{A. Measuring the fatigue damage of the penstock}

To assess the fatigue damage of the penstock, we use the head at each penstock element (simulated as described in Section IV) to compute the fatigue (subsection III.B-1) and finally apply the Miner's rule [4] to define, for each penstock element $i=1, \ldots, 50$, the cumulative damage $D_{i}$ :

$$
D_{i}=\sum_{j=1}^{J} \frac{n_{i, j}}{N_{i, j}}
$$

where $j$ denotes the set of cycles at a given stress level, $n_{i, j}$ is the cycle count from the rainflow algorithm, $N_{i, j}$ is the number of cycles to failure from the Wohler curve.

\section{B. Results}

We evaluate and compare the reduction of the fatigue damage achieved by the filters discussed in Section III against the case where no filter is applied. When no filter is applied, the HPP compensates for the difference between the grid frequency set-point $(50 \mathrm{~Hz})$ and frequency measurements $\left({ }^{1}\right)$. When a filter is applied, the plant compensates for the filtered frequency deviation. The cut-off frequency $f_{0}$ in (1) for our case study is $0.25 \mathrm{~Hz}$. We have first noted that $f_{0}$ achieves a substantial stress reduction compared to the aging-aware filter to the detriment, however, of the tracking performance of the original frequency signal. In the context of a hybrid plant with a BESS, worse transient tracking performance determines a higher energy capacity requirements because the BESS is required to compensate for longer variations of the power setpoint. In order to make results comparable, we adapt the cutoff frequency of the linear filter to achieve similar tracking performance (in the root mean square error sense) as the agingaware one, finally obtaining a value of $0.9 \mathrm{~Hz}$. After having tuned the cut-off frequency of the low-pass filter so as to attain similar tracking performance as the aging-aware one, it is possible to analyze and compare their impact on penstock fatigue, that is shown in Fig. 5. The y-axis of Fig. 5 refers to the relative damage index, that is calculated by dividing the cumulative damage (8) of each portion of the penstock by the largest cumulative damage incurred by the case with no filter. Fig. 5 shows that both filter leads a substantial reduction the aging thanks to smoothing the frequency measurements of the case without filter. The fatigue-aware filter achieves a substantial reduction of the damage index in all the penstock elements but one (i.e., the first element), where however the damage level scores low. In the parts of the penstock subject to the largest damage levels, (around the 5th element of the penstock), the low-pass filter achieves a relative damage index of 0.39 , whereas the aging-aware of 0.32 , thus reducing the fatigue damage by nearly $20 \%$.

\footnotetext{
${ }^{1}$ Frequency measurements are from [23] and refer to the European synchronous system.
}

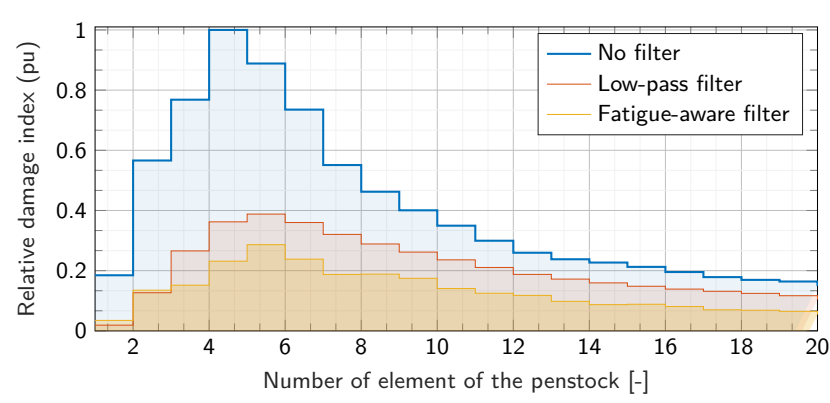

Fig. 5. Relative cumulative damage index along the penstock with reference to the case without filtering after fatigue-aware and low-pass filter action

\section{CONCLUSIONS}

We have considered the problem of reducing the penstock fatigue induced by PFC to the grid with a medium-head HPP. We have proposed two filters. The first is a first-order linear filter applied to the input power set-point to the plant. By reducing the control set-point variations, it reduces the water hammer effects and stress cycles conducive to penstock fatigue. The second filter, called fatigue-aware, leverages an explicit model of the fatigue to selectively remove from the input control signal those patterns that impact the most on penstock fatigue. The performance of the two filters was evaluated and compared considering a medium-head $230 \mathrm{MW}$ HPP power plant providing primary frequency control. Both filtering actions achieved to reduce the stress levels by five orders of magnitude. The fatigue-aware achieved better performance than the low-pass filter because it achieved lower fatigue levels while attaining similar tracking performance of the input control signal. Future works include validating results for high-head power plants, including surge tanks, and implementing the controller in a hybrid power plant considering a battery energy storage system.

\section{APPENDIX A \\ MOdel AND CONTROLLER VALIDATION}

We validate our Matlab implementation of the HPP model against SIMSEN, a commercial software for simulating hydropower plants. The validation includes evaluating relevant hydraulic quantities during: i) water hammer and ii) step response of the Francis turbine. The step response of the Francis turbine due to a change of 0.1 [p.u.] on the guide vane is shown in Fig. 7. The simulations shows the inverse response of head when the vane is closed, i.e. the flow starts reducing but the water head suddenly increases due to the smaller passage. The comparison shows a good matching between the two models. Fig. 6 shows the head in the penstock when suddenly closing the valve at $\mathrm{t}=1 \mathrm{~s}$ (i.e., water hammer). Our model shows similar behavior to the benchmark model.

We validate the controller's dynamic performance by verifying that we comply with the qualification test for primary frequency control [24]. It consists of ensuring that the plant, following a ramp variations of the frequency signal of $0.2 \mathrm{~Hz}$ over 10 seconds, delivers $50 \%$ and $100 \%$ of its total power 


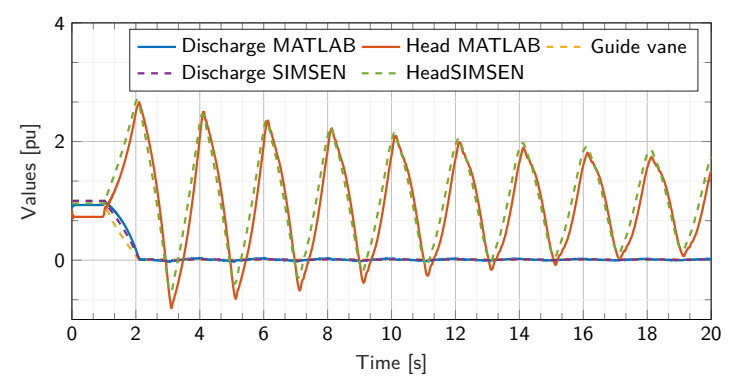

Fig. 6. Water hammer: heads and discharges at downstream valve resulting from a sudden closure of the valve at $t=1 \mathrm{~s}$.

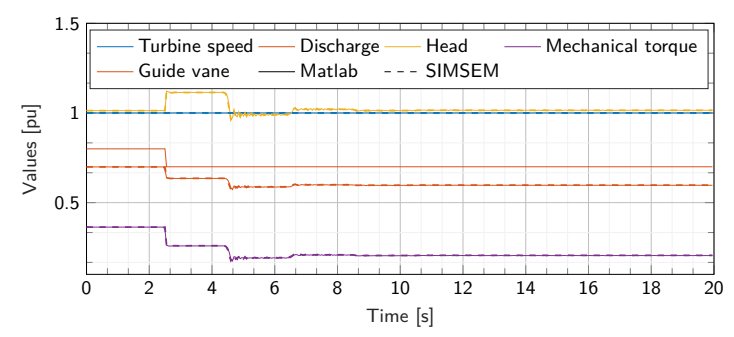

Fig. 7. Francis turbine's step response: heads, discharges and mechanical torques resulting from a guide vane step of 0.1 p.u. at $\mathrm{t}=2.5 \mathrm{~s}$.

output within 15 and 30 seconds, respectively, and it is within a specified envelope. Fig. 8 shows the results for a downward frequency ramp and shows the performance of the controller complies with the qualification test.

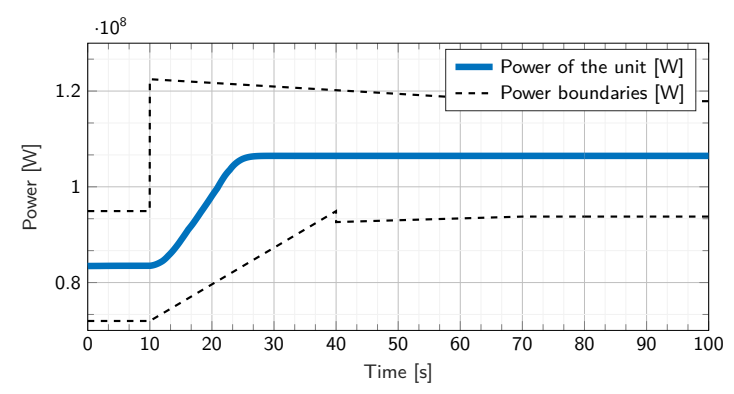

Fig. 8. Simulation results of active power time evolution resulting from PFR test case with frequency drop of $\Delta f=-200 \mathrm{mHz}$ and permanent speed droop $B_{s}=4 \%$

\section{REFERENCES}

[1] "Pan-European system with an efficient coordinated use of flexibilities for the integration of a large share of RES | EU-SysFlex Project | H2020 | CORDIS | European Commission." [Online]. Available: https://cordis.europa.eu/project/id/773505

[2] "Optimal System-Mix Of flexibility Solutions for European electricity | OSMOSE Project | H2020 | CORDIS | European Commission." [Online]. Available: https://cordis.europa.eu/project/id/773406

[3] "Hydropower Extending Power System Flexibility | XFLEX HYDRO Project | H2020 | CORDIS | European Commission." [Online]. Available: https://cordis.europa.eu/project/id/857832

[4] M. Dreyer, C. Nicolet, A. Gaspoz, D. Biner, S. Rey-Mermet, C. Saillen, and B. Boulicaut, "Digital clone for penstock fatigue monitoring," in IOP Conference Series: Earth and Environmental Science, vol. 405, no. 1. IOP Publishing, 2019, p. 012013.
[5] A. Borghetti, M. Di Silvestro, G. Naldi, M. Paolone, and M. Alberti, "Maximum efficiency point tracking for adjustable-speed small hydro power plant," in 16th Power Systems Computation Conference (PSCC'08), no. CONF, 2008.

[6] L. Fabietti, T. T. Gorecki, E. Namor, F. Sossan, M. Paolone, and C. N. Jones, "Enhancing the dispatchability of distribution networks through utility-scale batteries and flexible demand," Energy and Buildings, vol. 172, pp. 125-138, 2018.

[7] _ "Dispatching active distribution networks through electrochemical storage systems and demand side management," in 2017 IEEE Conference on Control Technology and Applications (CCTA). IEEE, 2017, pp. 1241-1247.

[8] O. Schmidt, A. Hawkes, A. Gambhir, and I. Staffell, "The future cost of electrical energy storage based on experience rates," Nature Energy, vol. 2, no. 8, pp. 1-8, 2017.

[9] Australian Energy Market Operator, "Initial operation of the Hornsdale power reserve battery energy storage system," 2018.

[10] M. Bozorg, F. Sossan, J.-Y. Le Boudec, and M. Paolone, "Influencing the bulk power system reserve by dispatching power distribution networks using local energy storage," Electric Power Systems Research, vol. 163 pp. 270-279, 2018.

[11] Y. Zuo, M. Paolone, and F. Sossan, "Effect of voltage source converters with electrochemical storage systems on dynamics of reduced-inertia bulk power grids," arXiv preprint arXiv:1910.05801, 2019.

[12] W. Yang, P. Norrlund, L. Saarinen, J. Yang, W. Zeng, and U. Lundin, "Wear Reduction for Hydropower Turbines Considering Frequency Quality of Power Systems: A Study on Controller Filters," IEEE Transactions on Power Systems, vol. 32, no. 2, pp. 1191-1201, Mar. 2017. [Online]. Available: https://ieeexplore.ieee.org/document/7514942/

[13] C. Nicolet, R. Berthod, N. Ruchonnet, and F. Avellan, "Evaluation of possible penstock fatigue resulting from secondary control for the grid," Proceedings of HYDRO, 2010.

[14] Y.-L. Lee and H.-T. Kang, "Chapter 9 - vibration fatigue testing and analysis," in Metal Fatigue Analysis Handbook. Boston: Butterworth-Heinemann, 2012, pp. 333 - 382. [Online]. Available: http://www.sciencedirect.com/science/article/pii/B9780123852045000094

[15] A. Pachoud, R. Berthod, P. Manso, and A. Schleiss, "Advanced models for stress evaluation and safety assessment in steel-lined pressure tunnels," International Journal on Hydropower and Dams, pp. 77-82, 09 2018.

[16] A. International, “ASTM E1049-85(2005), Standard Practices for Cycle Counting in Fatigue Analysis," Internet Requests for Comments, ASTM International, West Conshohocken, PA,, RFC 1654, 2005. [Online]. Available: http://www.astm.org/cgi-bin/resolver.cgi?E1049

[17] A. Niesłony, "Determination of fragments of multiaxial service loading strongly influencing the fatigue of machine components," Mechanical Systems and Signal Processing, vol. 23, no. 8, pp. 2712-2721, Nov. 2009.

[18] I. Hadley, "Bs 7910:2013 in brief," International Journal of Pressure Vessels and Piping, vol. 165, pp. 263 - 269, 2018. [Online]. Available: http://www.sciencedirect.com/science/article/pii/S0308016118302102

[19] P. Kundur and N. Balu, Power System Stability and Control, ser. EPRI power system engineering series. McGraw-Hill, 1994.

[20] E. De Jaeger, N. Janssens, B. Malfliet, and F. Van De Meulebroeke, "Hydro turbine model for system dynamic studies," IEEE Transactions on Power Systems, vol. 9, no. 4, pp. 1709-1715, 1994.

[21] C. Nicolet, "Hydroacoustic modelling and numerical simulation of unsteady operation of hydroelectric systems," Ph.D. dissertation, EPFL, Lausanne, 2007. [Online]. Available: http://infoscience.epfl.ch/record/98534

[22] C. Landry, C. Nicolet, J. Gomes, and F. Avellan, "Methodology to determine the parameters of the hydraulic turbine governor for primary control," Power Vision Engineering, Tech. Rep., 2019.

[23] M. Pignati, M. Popovic, S. Barreto Andrade, R. Cherkaoui, D. Flores, J.-Y. Le Boudec, M. M. Maaz, M. Paolone, P. Romano, S. Sarri, T. T Tesfay, D.-C. Tomozei, and L. Zanni, "Real-Time State Estimation of the EPFL-Campus Medium-Voltage Grid by Using PMUs," in The Sixth Conference on Innovative Smart Grid Technologies (ISGT2015), 2014.

[24] M. Scherer, D. Schlipf, and W. Sattinger, "Test for primary control capability,' Internet Requests for Comments, Swissgrid, RFC, 2011. [Online]. Available: http://www.swissgrid.ch 